\title{
Sunlight-driven Photocatalytic Degradation of Ciprofloxacin by Carbon Dots Embedded in ZnO Nanostructures
}

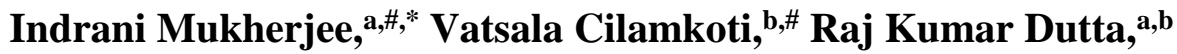 \\ ${ }^{a}$ Centre of Nanotechnology, Indian Institute of Technology, Roorkee, India 247667 \\ ${ }^{b}$ Department of Chemistry, Indian Institute of Technology, Roorkee, India 247667
}

\section{$\underline{\text { Supporting Information }}$}

\section{Supplementary results and discussion}

The effect of the concentration of $\mathrm{CDs}$ in the $\mathrm{ZnO} / \mathrm{CD}$ NCs on the efficiency of photocatalytic degradation of CIP solution has been studied by varying the initial amount of the CDs precursor, i.e., by taking $2.5 \mathrm{~mL}$ (batch denoted by $\mathrm{ZnO} / \mathrm{CD}_{2.5}$ ) and $7.5 \mathrm{~mL}$ (denoted by $\mathrm{ZnO} / \mathrm{CD}_{7.5}$ ), respectively (Fig.S1b). Photocatalytic degradation of CIP was studied following the same protocol as described in the main manuscript (i.e., $0.6 \mathrm{~g} \mathrm{~L}^{-1}$ photocatalyst dispersed in $50 \mathrm{~mL}$ of CIP solution at its natural $\mathrm{pH}$ 6.3). It can be observed that $\mathrm{ZnO} / \mathrm{CD}_{2.5}$ could degrade $90 \%$ of the drug within 110 min while $\sim 96 \%$ CIP was removed by $\mathrm{ZnO} / \mathrm{CD}_{7.5}$ within the same time interval. The $\mathrm{ZnO} / \mathrm{CD}_{2.5}$ contains a lesser concentration of $\mathrm{CDs}$ on the $\mathrm{ZnO}$ surface which reduced the effective electronhole separation of $\mathrm{ZnO}$ induced by $\mathrm{CDs}$, thereby causing a decline in the photocatalytic activity $(\mathrm{k}$ $=0.020 \mathrm{~min}^{-1}$ ). On the other, an increment of $\mathrm{CDs}$ content onto $\mathrm{ZnO}$ (i.e., $\mathrm{ZnO} / \mathrm{CD}_{7.5}$ ) caused a slight decrease in degradation rate to $0.028 \mathrm{~min}^{-1}$. Thus it can be inferred that a mere increase in CDs concentration will not always directly increase the photocatalytic efficiency of the catalyst. Instead, a higher amount of $\mathrm{CDs}$ on the $\mathrm{ZnO}$ surface will populate the active states, thereby reducing the contact area between the probe molecule and the photocatalyst. 
${ }^{1}$ Also, when present in large amounts, CDs tend to agglomerate, which can restrict the absorption of visible light by the photocatalyst ${ }^{2}$ and thus inhibit the generation of reactive oxygen species hindering the photocatalytic process to proceed. ${ }^{3}$ Hence, it is concluded that an optimum amount of CDs must be added to achieve the best photocatalytic performance (in our case it is $\mathrm{ZnO} / \mathrm{CD}_{5}$ ). In contrast, overloading of CDs causes a detrimental effect on the overall photocatalytic activity of the nanocomposite.

The band edge positions of $\mathrm{ZnO}$ was empirically calculated using the following expression

$4:$

$$
\begin{aligned}
& \mathrm{E}_{\mathrm{CB}}=\chi-\mathrm{E}^{\mathrm{e}}-0.5 \mathrm{E}_{\mathrm{g}} \\
& \mathrm{E}_{\mathrm{VB}}=\mathrm{E}_{\mathrm{CB}}+\mathrm{E}_{\mathrm{g}}
\end{aligned}
$$

where, $\mathrm{E}_{\mathrm{CB}}$ and $\mathrm{E}_{\mathrm{VB}}$ are the respective potentials of conduction band edge minima and valence band edge maxima, $\chi$ is the absolute electronegativity of the semiconductor that is calculated from the geometric mean of the electronegativities of the constituent atoms ( $\chi$ of $\mathrm{ZnO}$ is $5.79 \mathrm{eV}), \mathrm{E}^{\mathrm{e}}(4.5$ $\mathrm{eV}$ ) is the energy of free electrons on the standard hydrogen scale (NHE scale) and $\mathrm{E}_{\mathrm{g}}$ is the band gap energy of the semiconductor. Taking $\mathrm{E}_{\mathrm{g}}$ of $\mathrm{ZnO}$ as $3.31 \mathrm{eV}$, the values of $\mathrm{E}_{\mathrm{CB}}$ and $\mathrm{E}_{\mathrm{VB}}$ of $\mathrm{ZnO}$ were determined as $2.95 \mathrm{eV}$ and $-0.36 \mathrm{eV}$, respectively. 


\section{Supplementary Figures}

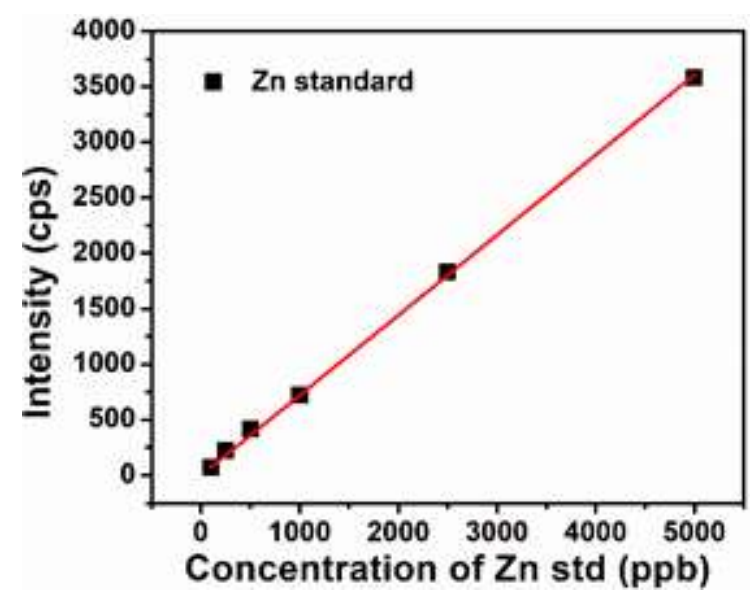

Figure S1. Calibration plot by ICP-OES
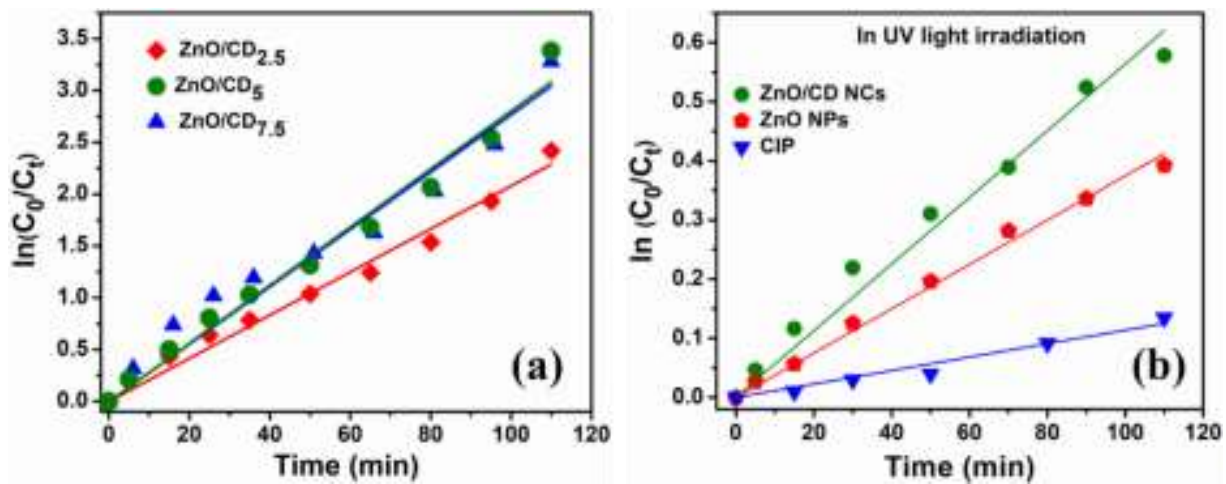

Figure $\mathrm{S} 2$. The plot of $\ln \left(\mathrm{C}_{0} / \mathrm{C}_{\mathrm{t}}\right)$ vs. irradiation time of CIP degradation, (a) by ZnO/CD NCs with varying amount of $\mathrm{CDs}$, (b) by $\mathrm{ZnO} / \mathrm{CD} \mathrm{NCs}$ and $\mathrm{ZnO}$ NPs under UV light irradiation 

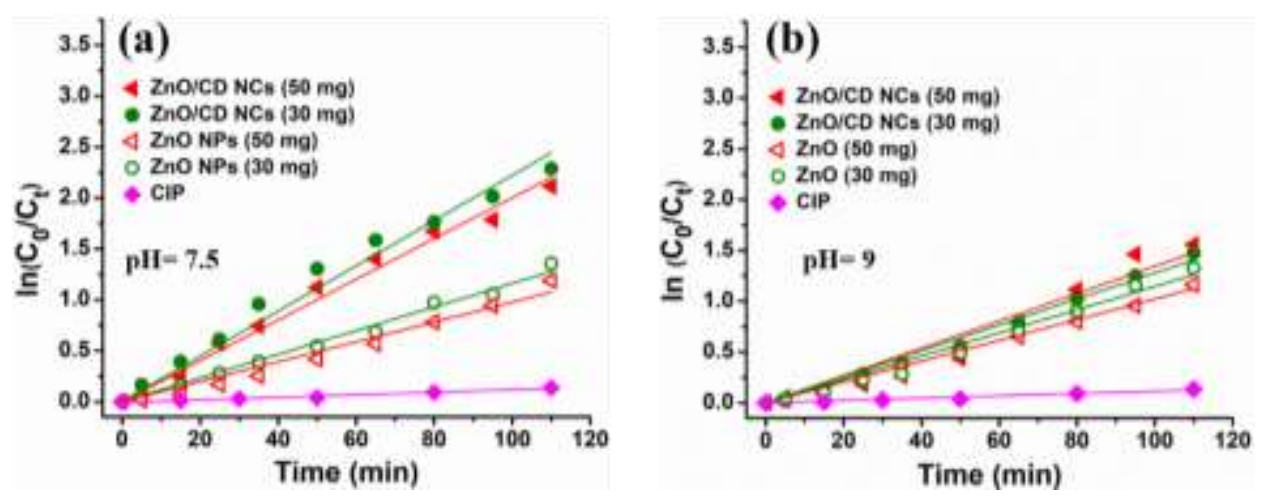

Figure S3. plot of $\ln \left(C_{0} / C_{t}\right) v s$. irradiation time of CIP degradation by the corresponding photocatalysts at $\mathrm{pH}=7.5$ and 9 , respectively.

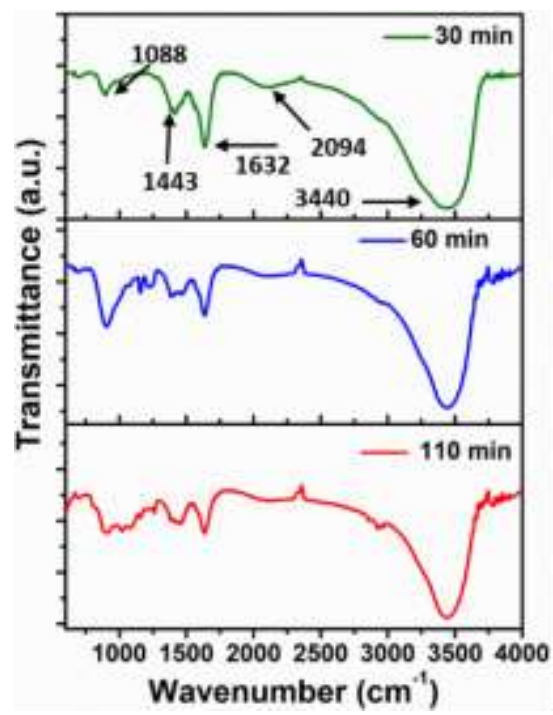

Figure S4. FTIR spectra of the ZnO/CD NCs photocatalyst during the course of the photocatalytic experiment 
References

\footnotetext{
${ }^{1}$ Wang, Y.; Chen, J.; Xu, Q.; Li, Y.; Fu, T.; Jiang, G.; Li, Y.; Zhao, Z.; Wei, Y. Novel visiblelight-driven S-doped carbon dots/BiOI nanocomposites: improved photocatalytic activity and mechanism insight. J. Mater. Sci. 2017, 52, 7282-7293.

${ }^{2}$ Di, J.; Xia, J.; Ji, M.; Xu, L.; Yin, S.; Zhang, Q.; Chen, Z.; Li, H. Carbon quantum dots in situ coupling to bismuth oxyiodide via reactable ionic liquid with enhanced photocatalytic molecular oxygen activation performance. Carbon 2016, 98, 613-623.

${ }^{3}$ Li, H.; Liu, R.; Liu, Y.; Huang, H.; Yu, H.; Ming, H.; Lian, S.; Lee, S.; Kang, Z. Carbon quantum dots $/ \mathrm{Cu}_{2} \mathrm{O}$ composites with protruding nanostructures and their highly efficient (near) infrared photocatalytic behavior. J. Mater. Chem. 2012, 22, 17470-17475.

${ }^{4}$ Naseri, A.; Samadi, M.; Mahmoodi, N.; Pourjavadi, A.; Mehdipour, H.; Moshfegh A.

Tuning composition of Electrospun $\mathrm{ZnO} / \mathrm{CuO}$ nanofibers: toward controllable and efficient solar photocatalytic degradation of organic pollutants. J. Phys. Chem. C 2017, 121, 3327-3338.
} 